\section{Mzf1 controls cell proliferation and tumorigenesis}

\author{
Mirella Gaboli, 1,2,5 Paraskevi A. Kotsi, ${ }^{1,2,5}$ \\ Carmela Gurrieri, ${ }^{1,2}$ Giorgio Cattoretti, ${ }^{3}$ \\ Simona Ronchetti, ${ }^{1,2}$ Carlos Cordon-Cardo, ${ }^{2}$ \\ Hal E. Broxmeyer, ${ }^{4}$ Robert Hromas, ${ }^{4}$ and \\ Pier Paolo Pandolfi ${ }^{1,2,6}$
}

\begin{abstract}
${ }^{1}$ Molecular Biology Program, ${ }^{2}$ Department of Pathology Memorial Sloan-Kettering Cancer Center, Sloan-Kettering Division, Graduate School of Medical Sciences, Cornell University, New York, New York 10021, USA; ${ }^{3}$ Columbia University College of Physicians and Surgeons, New York, New York 10032, USA; ${ }^{4}$ Department of Medicine (Hematology/Oncology) and Microbiology/Immunology, Indiana University Medical Center, and the Walther Cancer Institute, Indianapolis, Indiana 46202-5121, USA.
\end{abstract}

\begin{abstract}
MZF1 is a transcription factor belonging to the Krüppel family of zinc finger proteins, expressed in totipotent hemopoietic cells as well as in myeloid progenitors. Here we have inactivated Mzfi1 by gene targeting. $\mathrm{Mzf1}^{-/-}$ mice develop lethal neoplasias characterized by the infiltration and complete disruption of the liver architecture by a monomorphic population of cells of myeloid origin reminiscent of human chloromas. Mzf1 inactivation results in a striking increase of the autonomous cell proliferation and of the ability of $\mathrm{Mzf1}^{-/-}$hemopoietic progenitors to sustain long-term hemopoiesis. These findings demonstrate that Mzf1 can act as a tumor/ growth suppressor in the hemopoietic compartment.
\end{abstract}

Received April 17, 2001; revised version accepted May 9, 2001.

Zinc finger proteins play a crucial role in regulating normal hemopoiesis (Shivdasani and Orkin 1996). Furthermore, several genes encoding zinc finger transcription factors are involved in chromosomal translocations associated with hemopoietic malignancies (Look 1997), and their functional deregulation or inactivation is an essential step in leukemogenesis (He et al. 2000 and references therein). Myeloid zinc finger 1 (MZF-1) is a transcription factor of the Krüppel family of zinc finger proteins originally cloned from a cDNA library from a patient with chronic myeloid leukemia (Hromas et al. 1991). Within the hemopoietic compartment MZF-1 expression is restricted to totipotent bone marrow (BM) progenitor cells and early myeloid progenitors and precursors, and is not detectable in fully differentiated blood

[Key Words: MZF; hematopoiesis; tumorigenesis; knockout mice; myeloid progenitors]

${ }^{5}$ These authors contributed equally to this work.

${ }^{6}$ Corresponding author.

E-MAIL p-pandolfi@ski.mskcc.org; FAX (212) 717-3374.

Article and publication are at http://www.genesdev.org/cgi/doi/10.1101/ gad.902301. cells (Bavisotto et al. 1991). MZF-1 contains 13 zinc finger domains divided into two groups, which can bind DNA independently of each other (Morris et al. 1994; Hromas et al. 1996). In vitro, in transient transfection experiments, MZF-1 can activate transcription in cells of hemopoietic origin, and it has also been found to repress transcription in nonhemopoietic cells (Morris et al. 1995; Hromas et al. 1996). MZF1 antisense oligonucleotides inhibit granulocyte colony-stimulating factor (G-CSF)-driven granulocyte colony formation (Bavisotto et al. 1991). However, MZF1 overexpression in embryonic stem (ES) cells also appears to interfere with the ability of these cells to undergo hemopoietic commitment as well as erythromyeloid colony formation (Perrotti et al. 1995). These data may suggest a role for MZF1 in the control of myeloid differentiation. However, the biological function of MZF1 is presently unknown, also in view of the seemingly contradictory nature of these observations.

Here we show, in vivo, in knockout $(\mathrm{KO})$ mice, that Mzf1 controls the proliferative potential of hemopoietic cells acting as a growth and tumor suppressor.

\section{Results and Discussion}

Generation of $\mathrm{Mzf1}^{-/-}$mice

We characterized and ablated the murine $M z f 1$ gene by homologous recombination. Sequencing of the Mzf1 coding region, which is retained in a single exon, revealed an $87.5 \%$ identity and a $97.2 \%$ similarity between the human and mouse proteins. The murine $M z f 1$ gene was found to be expressed in BM cells, but also in adult brain, testis, keratinocytes, and thymus (not shown). Using a targeting vector for positive/negative selection in mouse ES cells, we replaced the Mzf1 coding region with the positive selectable marker (neomycin cassette), completely ablating the region encoding the Mzf1 DNAbinding domain, and obtained several $\mathrm{Mzf1}^{+/-}$ES cell clones. $\mathrm{Mzf1^{-1- }}$ mutants in a pure $129 \mathrm{~Sv}$ background were generated from 4 of 27 independently targeted ES cell clones (Fig. 1A-C; Materials and Methods). $\mathrm{Mzf1}^{-{ }^{-}}$ mutants were born at Mendelian frequencies and were developmentally normal (not shown). The disruption of $\mathrm{Mzf1^{-1 }}$ - was confirmed by RT-PCR analysis on RNA from BM cells (Fig. 1D; Materials and Methods).

Progressive accumulation of myeloid cells in the bone marrow from $\mathrm{Mzf}^{-/-}$mice

We characterized myeloid and lymphoid hemopoiesis in $\mathrm{Mzf1^{-1- }}$ mice and control littermates. Analysis of peripheral blood (PB) cell populations with the use of an automated counter (Technicon H2), and by differential counts performed on Wright-Giemsa-stained smears, did not reveal significant differences between $\mathrm{Mzf1}^{-/-}$mice and wild-type sex-matched syngeneic littermates (not shown; Materials and Methods). Spleen, BM, lymph nodes, and thymus from $\mathrm{Mzf1}^{-/}$and wild-type mice were then analyzed by differential counts and by flow cytometry with antibodies specific for hemopoietic stem cells/progenitors, myeloid cells, T- and B-lymphocytes. No significant differences were found in the lymphoid 


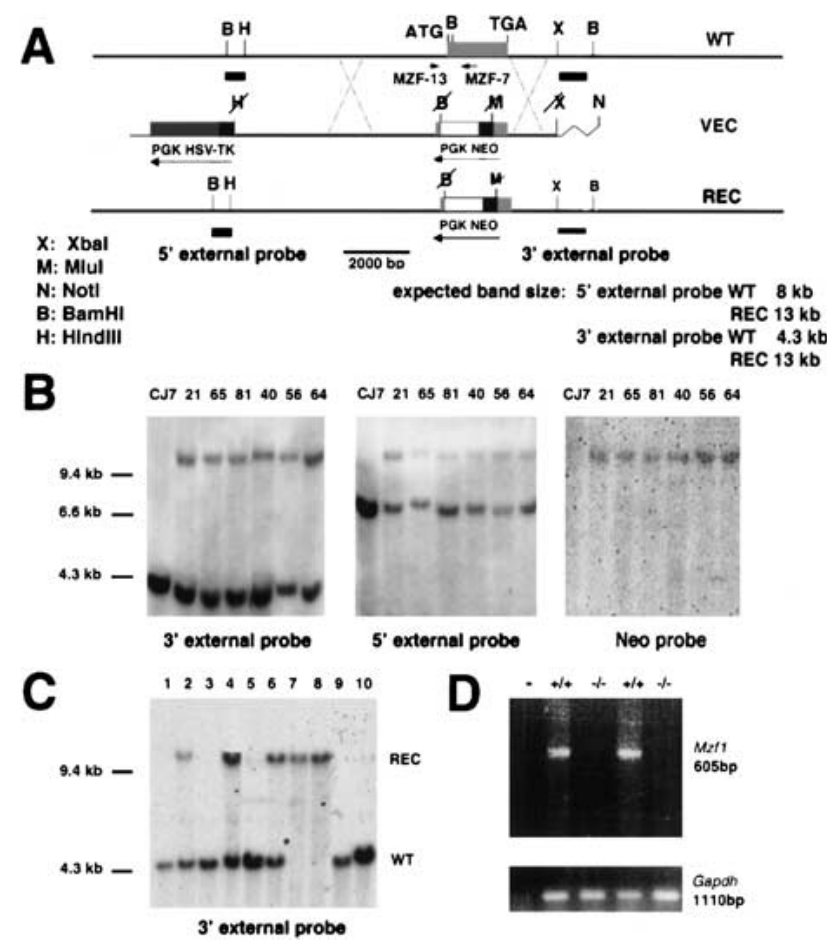

Figure 1. Targeted disruption of Mzf1 in the mouse germ line. (A) Maps of the wild-type Mzf1 locus (WT, top), the targeting vector (VEC, middle), and the predicted targeted gene (REC, bottom). The Mzf1 genomic sequence is depicted as a thick line with a gray box representing the Mzf1 coding sequence. Sequences from the PNT plasmid (Tybulewicz et al. 1991) are shown as thin lines, with an open box representing the neomycin resistance cassette (Neo), a dark gray box representing the herpes simplex virus thymidine kinase cassette (HSV-TK), and the filled black boxes representing the PGK promoters driving the expression of the HSV-TK and Neo genes. The Neo and $M z f 1$ genes are transcribed in opposite directions. The Mzf1 genomic fragments used as probes for Southern blot analysis are indicated $\left(5^{\prime}\right.$ external probe and $3^{\prime}$ external probe). (B) BamHI; (H) HindIII; (X) XbaI restriction sites are shown. (B) Southern blot analysis of CJ7 untransfected cells and recombined clones after digestion with BamHI and hybridization with the three indicated probes. $(C)$ Southern blot of mouse tail DNAs from one litter obtained by crossing $M z f 1$ heterozygous mice after digestion with BamHI. (D) RT-PCR analysis using oligonucleotides MZF-13 and MZF-7 confirming the complete inactivation of the Mzf1 locus in BM cells. Amplification with a Gapdh primer pair was used to normalize for the amount of cDNA.

compartment (not shown; Materials and Methods). However, we observed a progressive and consistent accumulation of the mature Mac- $1^{+}$(Sca- 1 and Gr-1 negative) myeloid population in the BM of $\mathrm{Mzf1}^{-/-}$mice compared to syngeneic sex, age matched wild-type controls (increase ranging between $5 \%$, at 1 mo of age, to $10 \%$ in 10-month-old mutants; 10 mutants and 10 wildtype controls were analyzed; mean difference $8.7 \%$; $P<.001$ ) (Fig. 2A). Thus, unexpectedly, at the steady state, Mzf1 inactivation does not impair the ability of myeloid and lymphoid cells to terminally differentiate, but affects the size of the Mac- $1^{+}$myeloid compartment in the BM.
$\mathrm{Mzf1}^{-/-}$mice develop lethal myeloid neoplasias characterized by the infiltration and complete disruption of the liver architecture

To determine whether the inactivation of $M z f 1$ would result in overt disease, mice were followed throughout their life (during a 30-mo follow-up period). One group of mice was bled on a monthly basis, together with agematched littermate controls, and sacrificed when developing manifest signs of disease (e.g., ascites; see below), or when hemoglobin levels dropped to $<8 \mathrm{~g} / \mathrm{dL}$. Automated and differential counts, as well as morphological analysis of PB cells, were performed on each sample. In a second group, $\mathrm{Mzf1}^{-/-}$and wild-type controls were aged,
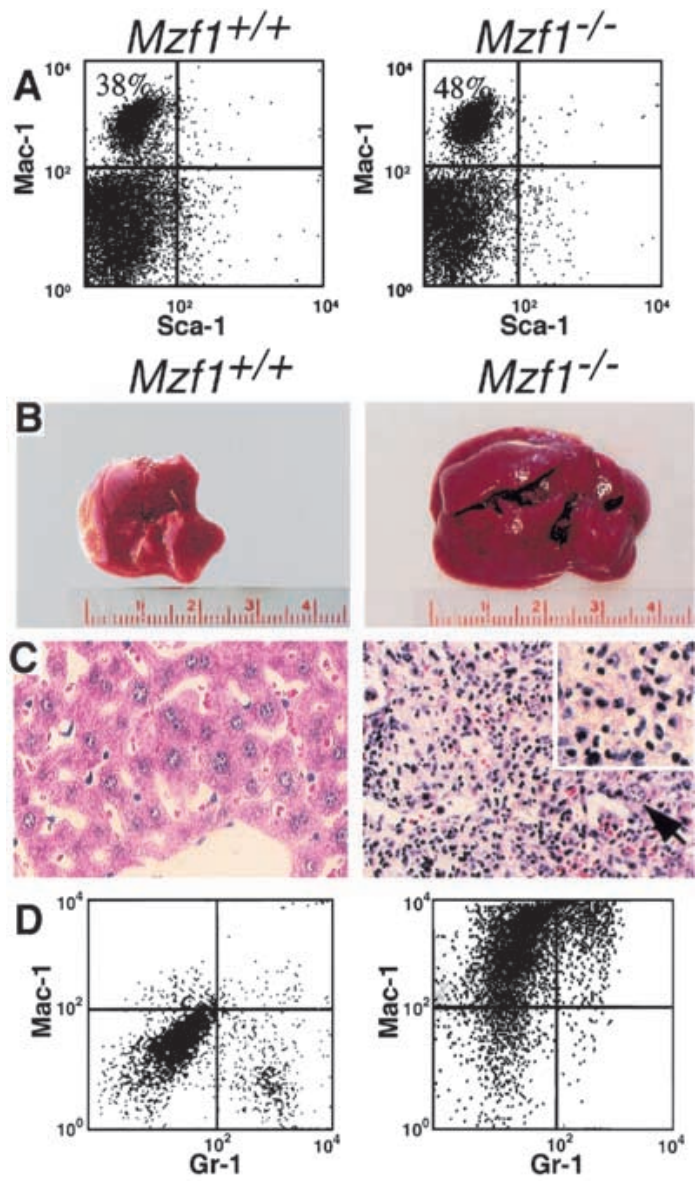

Figure 2. Accumulation of myeloid cells and hepatic neoplasias in $\mathrm{Mzf1}^{-1-}$ mice. (A) Flow cytometry analysis of BM cells in 10-month-old mice, stained with Mac-1 and Sca-1 antibodies. The genotype of the mice is indicated. At this age, $\mathrm{Mzf1}^{-/-}$mice did not show signs of overt disease. $(B-D)$ Hepatic neoplasias in $M z f 1^{-/-}$mice: wild-type (left) and diseased $\mathrm{Mzf1}^{-{ }^{--}}$mice (right). (B) Macroscopic illustration of liver. The liver of $\mathrm{Mzf1}^{-/-}$mouse is markedly enlarged in view of the infiltration of the neoplastic population. (C) Histopathological analysis of liver sections stained with hematoxylin and eosin (original magnification, 400x): The liver architecture is effaced by the dramatic infiltration of a monomorphic blast population. The black arrow indicates a residual hepatocyte. (D) Flow cytometric analysis of liver cells stained with Mac-1 and Gr-1 antibody. Mac-1 positive cells (upper left quadrant) infiltrate the diseased liver from a $\mathrm{Mzf1}^{-/-}$ mutant. 
and four from each genotype sacrificed every month for gross and microscopic examination of all organs, in particular PB, BM, spleen, liver, lymph nodes, and thymus. Cell populations in these organs were analyzed morphologically on smears, touch preparations, and cytospin preparations stained with Wright-Giemsa. By year 2, we observed extramedullary hemopoiesis in the liver or spleen in all $\mathrm{Mzf1}^{-/}$mice analyzed (28 of 28 older than $2 \mathrm{yr})$. Extramedullary hemopoiesis affecting the spleen, but never the liver, was observed only in 5 of the 36 wild-type mice older than 2 yr (not shown). Of the $M z f 1^{-1-}$ mice, 30\% $(n=9)$ developed a peculiar neoplastic disease affecting the liver and the spleen of which they succumbed between 24 to 32 mo. In these mice a monomorphic blastic cell population completely effaced the liver architecture (Fig. 2B,C). The morphological features of these cells were characteristic of hemopoietic blast with prominent nuclei. In some cases the neoplastic lesion was infiltrating the adjacent organs (i.e., the intestine). These neoplasias were highly reminiscent of solid tumor composed of myeloid cells. Surprisingly, the BM was never infiltrated by the neoplastic population (data not shown). However, the BM of $M z f 1^{-1-}$ mice was almost invariably hypercellular. In one case the PB was affected, but even in this case the BM was normal (blasts $<10 \%)$. Four (14\%) Mzf1 ${ }^{-/-}$mice developed high-grade B-cell lymphomas. Of the 36 wild-type mice analyzed, four developed lymphomas (11\%). None of the wild-type mice developed the liver/spleen neoplasia.

To define the histological origin and the proliferative properties of the neoplastic cell population, we performed flow cytometry and immunohistochemistry analysis. The infiltrating cells were: (1) Mac-1 positive. Mac-1 (CD11b) is expressed either in macrophages/ monocytes or in a very immature myeloid precursor (Fig. 2D); (2) positive for GM-colony-stimulating factor receptor alpha (GM-CSFR $\alpha)$, a marker also expressed on early myeloid progenitors (Fig. 3A-C); (3) S100 negative. S100 is a marker normally expressed on mature histiocytes (not shown); (4) strongly positive for the Ki67 proliferation marker (Fig. 3D-F). Leukemic cells from $M z f 1^{-1-}$ mice were transplantable in $129 /$ Sv syngeneic mice sublethally irradiated (500 Gy) (Material and Methods).
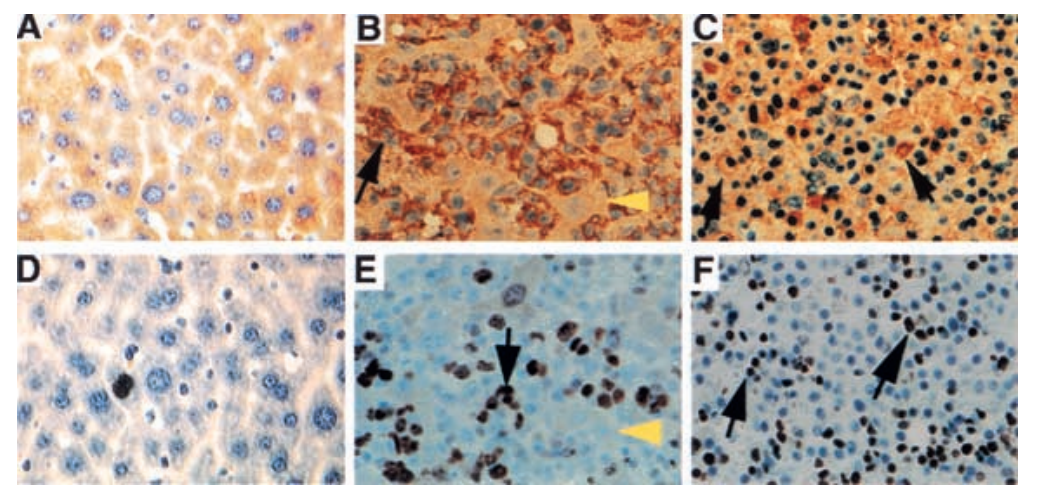

Figure 3. Neoplasias in $\mathrm{Mzf1^{-/- }}$ mice are made by highly proliferating myeloid cells. Immunohistochemical analysis of liver sections using anti-GM-CSFR $\alpha$ antibody (top) and anti-Ki-67 antibody (bottom). $(A, D)$ Wild-type and $(B, C, E, F)$ diseased $\mathrm{Mzf1}^{-1-}$ mice. Black arrows indicate positively stained cells and yellow arrowheads, unstained residual hepatocytes. Original magnifications, $(A, B, D, E) 400 \times ;(C, F) 200 \times$. Two different magnifications of the tumors are shown.
Thus, Mzf1 inactivation leads to the development of lethal tumors made of highly proliferating myeloid blasts, neoplasias that are reminiscent of human chloromas (see below; Harrison 1987).

\section{Increased number of progenitor cell-derived colonies from the bone marrow and spleen of $\mathrm{Mzf1}^{-/-}$mice}

To unravel the mechanisms by which Mzf1 acts as tumor suppressor, we then assessed the cell autonomous hemopoietic potential of $\mathrm{Mzf1}^{-/-} \mathrm{BM}$ progenitors before the occurrence of the neoplasias in mice of $12-16 \mathrm{wk}$ of age. To this end, we performed in vitro cultures of BM cells in standard cytokine and growth factor concentrations, from $\mathrm{Mzf1}^{-/-}$and control mice (Materials and Methods). Mzf1 inactivation resulted in a marked increase of both myeloid and erythroid colonies: colonyforming unit-granulocyte, macrophage (CFU-GM), burstforming unit-erythroid (BFU-E), and colony-forming unit-granulocyte, erythroid, macrophage, megakaryocyte (CFU-GEMM), from both the BM and the spleen (Fig. 4). Thus, under these concentrations of cytokines, $M z f 1^{-1-} \mathrm{BM}$ and spleen progenitors possess an increased clonogenic capacity.

\section{Increased proliferation of $\mathrm{Mzf1}^{-/-}$hemopoietic progenitor cells}

To determine whether the greater numbers of BM- and spleen-derived colonies obtained from $\mathrm{Mzf1}^{-/-}$mutants are the result of an increased proliferative potential of hemopoietic progenitor cells, we performed $\left[{ }^{3} \mathrm{H}\right]$ thymidine suicide assays. This established procedure allows the estimation of the percent of progenitors in S-phase: incorporation of $\left[{ }^{3} \mathrm{H}\right]$ thymidine into DNA takes place in actively proliferating cells resulting in cell death (Material and Methods; Maze et al. 1992). Thus, in this assay an increased proliferative potential of progenitor cells results in a reduced number of hemopoietic colonies. The BM and spleen cells from $\mathrm{Mzf1}^{-1-}$ and wild-type mice were cultured in the absence or presence of highspecific activity $\left[{ }^{3} \mathrm{H}\right]$ thymidine as a pulse exposure. After removal of $\left[{ }^{3} \mathrm{H}\right]$ thymidine, cells were plated in methylcellulose, in the presence of growth factors, to trigger the formation of hemopoietic colonies. $\mathrm{Mzf1^{-/- }}$ inactivation dramatically enhanced the proliferative capacity of hemopoietic progenitors and, in turn, their responsiveness to the hemopoietic growth factors present in the medium (Fig. 5A,B). As previously mentioned, in vitro, Mzf1 is known to regulate c-Myb expression at the transcription level. However, the effects on cell proliferation observed in $\mathrm{Mzf1^{-/- }}$ hemopoietic cells were not caused by an aberrant c-Myb expression. In fact, the levels of c-Myb were never found increased in $\mathrm{Mzf1}^{-/-}$mutants in both Western blot and TaqMan analyses performed on total BM cells or sorted early hemopoietic progenitors (not shown; Materials and Methods).

Increased long-term proliferative potential of $\mathrm{Mzf1}^{-/-}$hemopoietic progenitors

We then investigated whether the proliferative advantage of $\mathrm{Mzf1}^{-/-}$hemopoietic pro- 
A

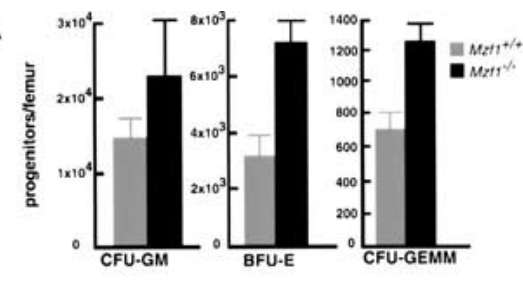

B

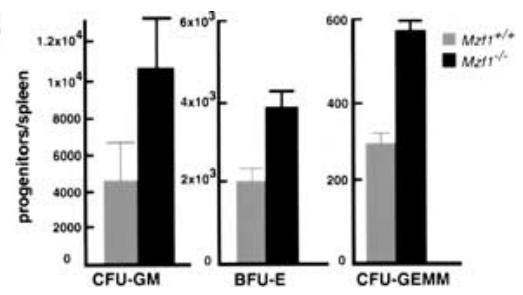

Figure 4. Increased numbers of progenitor-derived colonies in $\mathrm{Mzf1}^{-/-}$mice. The clonogenic potential of $\mathrm{Mzf1}^{-/-}$hemopoietic progenitors was evaluated in a methylcellulose colony-forming assay by scoring CFU-GM, BFU-E, and CFU-GEMM obtained from $(A)$ $\mathrm{BM}$ cells and $(B)$ spleen cells and expressing these results per organ. The values are averages \pm SE calculated from triplicates of one representative experiment carried out with five $\mathrm{Mzf1}^{-/-}$(black bars) and five syngeneic 129Sv control littermates (gray bars), plated as independent cultures.

genitors would result in an increased ability to support long-term hemopoiesis by performing long-term BM liquid cultures from $M z f 1^{-1-}$ mice and controls littermates (Material and Methods). From these cultures, clonogenic assays in standard cytokines conditions were carried out on a weekly basis. A much greater number of CFU-GM was produced from $M z f 1^{-1-}$ BM cells throughout the 4-wk culture (Fig. 5C). Therefore, Mzf1 inactivation also augments the ability of progenitor cells to support longterm hemopoiesis.

In summary, the present study leads to three major conclusions:

1. Mzf1 is dispensable for myeloid/hemopoietic differentiation at the steady state. Although in vitro antisense experiments have shown that MZF1 might control myeloid hemopoietic differentiation (Bavisotto et al. 1991; Perrotti et al. 1995), the inactivation of $M z f 1$ in vivo in the mouse did not affect this process, at least at the steady state, but resulted instead in an accumulation of BM Mac- $1^{+}$myeloid cells. In this respect, it is also worth noting that with gene targeting, the $M z f 1$ region coding for the DNA-binding domain was entirely deleted, thus resulting in the complete inactivation of $M z f 1$, including the Mzf1 isoform termed MZF1B or Mzf2 (Murai et al. 1997, 1998; Sander et al. 2000).

2. Mzf1 plays a crucial role in the negative regulation of the proliferative capacity of hemopoietic progenitors. Cells from the BM and spleen of $\mathrm{Mzf1}^{-/-}$mice yielded a greater number of colonies of all hemopoietic lineages in in vitro cultures. This may be explained either by an increased proliferation or by an increased commitment of progenitor cells to differentiate in response to the cytokines and growth factors present in the cultures. However, $\left[{ }^{3} \mathrm{H}\right]$ thymidine suicide assays show that Mzf1 inactivation results in a marked increase in the proportion of hemopoietic progenitors that are actively cycling at a given time, both in the bone marrow and in the spleen. Furthermore, this increased proliferative rate allows self-renewal of progenitors, as demonstrated by the fact that $M z f 1^{-/-}$BM cells gave rise to a greater number of colonies even after long-term BM culture. In contrast, analysis of apoptosis in early hemopoietic progenitor cells as evaluated by terminal deoxynucleotide end-labeling (TUNEL) and Annexin V assays did not reveal any significant difference between $\mathrm{Mzf1}^{-/-}$and wild-type mice (data not shown; Materials and Methods).

3. Mzf1 acts as growth and tumor suppressor in the myeloid hemopoietic compartment suggesting that Mzf1 inactivation or deregulated function could participate in tumorigenesis by lending a proliferative advantage to the neoplastic cells. From this point of view, it is intriguing that the human MZF1 gene is one of the most subtelomeric genes described so far located only a few kilobases from the subtelomeric repeat region of $19 \mathrm{q}$, which may lead to $M Z F 1$ loss as a consequence of telomeric erosion (Hoffman et al. 1996). The incidence of myeloid neoplasia in $\mathrm{Mzf1}^{-/-}$mice is higher
A

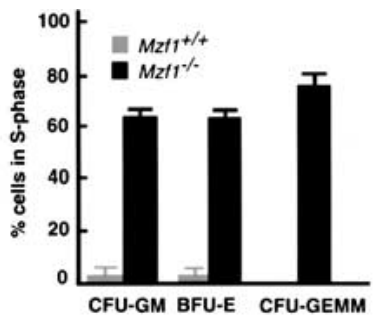

B

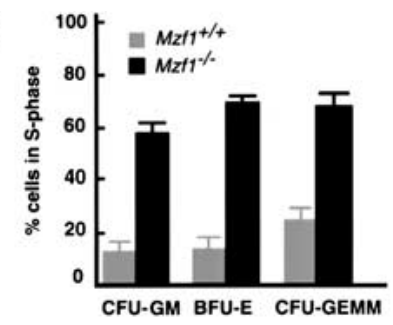

C

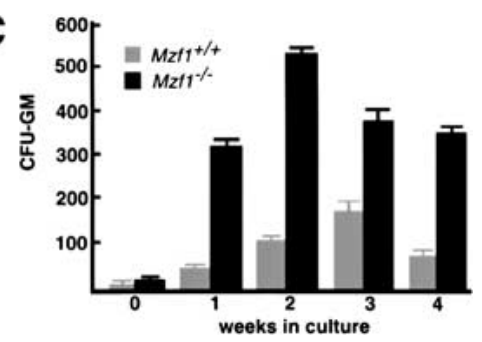

Figure 5. Increased cell cycling potential of hemopoietic progenitors and increased numbers of myeloid progenitor-derived colonies in long-term BM cultures from $M z f 1^{-/-}$mice. (A) The proportion of hemopoietic progenitors in S-phase, evaluated with the $\left[{ }^{3} \mathrm{H}\right]$ thymidine suicide assay, and calculated as the reduction in CFU-GM, BFU-E, and CFU-GEMM formation upon treatment with $\left[{ }^{3} \mathrm{H}\right]$ thymidine as opposed to cold thymidine in BM and $(B)$ spleen cells. The values are averages \pm SE calculated from triplicates of one experiment representative of two carried out with five $M z f 1^{-/-}$(black bars) and five syngeneic 129Sv control littermates (gray bars), plated as independent culture. $(C)$ Long-term BM culture in $\mathrm{Mzf1}^{-/-}$mice. The number of CFU-GM colonies obtained from BM cells after the indicated weeks of culture is shown. The values are averages \pm SE calculated from triplicates of one representative experiment carried out with BM cells from five $\mathrm{Mzf1}^{-/-}$(black bars) and five syngeneic 129Sv control littermates (gray bars), plated as independent cultures. 
than the incidence of myeloid leukemia in transgenic mice harboring potent oncogenes such as the PML$\mathrm{RAR} \alpha$ fusion gene of acute promyelocytic leukemia (only $20 \%$ of these transgenic mice develop leukemia after a long latency; He et al. 1997). However, the fact that $\mathrm{Mzf1}^{-/-}$mice are not born with cancer and that, on the contrary neoplasias in $\mathrm{Mzf1}^{-/-}$mice occur after a latency period strongly suggests that additional mutations are needed for full-blown transformation. The progressive expansion of the hemopoietic compartment within the BM, liver, and spleen as a result of Mzf1 inactivation would then favor the occurrence of additional transforming events. Mzf1 inactivation could dictate the peculiar hepatic and splenic localization of these neoplasias by modulating the expression of adhesion molecule that would retain the blasts within these organs. These findings prompt investigating whether MZF1 mutations or loss of heterozygosity occur in human hemopoietic tumors: myeloproliferative syndromes and myeloid leukemias are possible candidates. However, it is also worth noticing that malignant myeloid tumors localized to both soft tissues such as the liver and the bones (also known as chloromas; Harrison 1987) have been described and are, in this respect, straightforward candidate for this analysis.

\section{Materials and methods}

Generation of $\mathrm{Mzf1}^{-/-}$mice

To characterize the mouse $M z f 1$ genomic locus, we screened a $129 \mathrm{~Sv}$ mouse $\lambda$-phage genomic library (Stratagene) with a human MZF1 cDNA probe encoding the acidic non-zinc finger $\mathrm{N}$-terminal domain of the protein. Two overlapping clones were obtained. The coding sequence was determined by restriction enzyme mapping, DNA sequencing, and PCR. The targeting vector contains PGK-HSV-TK and PGK-NEO cassettes, the latter of which replaces, in the opposite orientation, the 1100-bp BamHIMluI fragment of $M z f 1$ coding region (which codes for almost the entire DNA-binding domain of Mzfl and its Mzf2 isoform: the first 11 zinc finger domains) and is flanked by Mzf1 genomic DNA (5' arm, 7.0-kb HindIII-BamHI fragment, 3' arm, 2.0-kb MluI-XbaI fragment). In addition, the presence of the PGK-NEO cassette within $M z f 1$ coding region introduces stop codons in all three frames, thereby interrupting the translation of the remaining two C-terminal zinc finger domains of the Mzf1 protein. The NotI linearized vector was electroporated into CJ7 ES cells and transfectants were selected in G418 $(350 \mu \mathrm{g} / \mathrm{mL})$ and Gancyclovir $(2$ $\mu \mathrm{M})$. Resistant clones were screened by Southern blotting with the $3^{\prime}$ external probe using a BamHI digestion. Recombined ES cell clones were further characterized by Southern blotting with $5^{\prime}$ external and internal NEO probes, using a BamHI digestion. Twenty-six mutant CJ7 ES clones heterozygous for the $\mathrm{Mzf1}$ deletion were obtained. $\mathrm{Mzf1^{-/- }}$ mutants were generated from four independently targeted ES cell clones according to standard procedures (Barna et al. 2000). Inactivation of the gene was confirmed by RT-PCR analysis. To this end, total BM RNA from 6- to 8-week-old mice was prepared using TRIzol (GIBCO BRL). RT-PCR was performed with $2 \mu \mathrm{g}$ of DNase I-treated RNA, by using cDNA Cycle Kit for RT-PCR (Invitrogen), according to the manufacturer's protocol. Two microliters of the synthesized cDNA was used for PCR with the following primers: sense MZF-13, 5'-TGCCTCACCAACCAGTTCCAA-3'; anti-sense MZF-7, 5'-CTGCGCACGAAGCCCTGGCCA- ${ }^{\prime}$. The quality of cDNA was accessed by amplification with Gapdh control primers: sense 5'-GTGAAGGTCGGTGTGAACGGA-3'; antisense 5'-TTATTA TGGGGGTCTGGGATGGAA- 3 '. The specificity of amplification was evaluated by hybridizing the PCR products, upon transfer on a nylon membrane, with internal primers (not shown).

Analysis of hemopoiesis in $\mathrm{Mzf1}^{-/-}$mice

Animals were analyzed throughout life along with littermate controls at bimonthly intervals (at least four mice from each genotype per time point). Mice were bled by retroorbital venipuncture. Leukocyte, platelet, and red cell counts were performed with an automated counter (Technicon H2). Differential counts of myeloid and lymphoid subpopulations were carried out on PB smears stained with Wright-Giemsa. Differential counts of three or four smears per animal were scored for a total of at least 300 cells. Single-cell suspensions from BM, spleen, lymph nodes, liver, and thymus were analyzed on a five-color FACStar Plus flow-cytometer (Beckton Dickinson, Mountain View, CA). Fluorochrome-conjugated antibodies for flow cytometry were CD45R/B220, CD34, CD43, Ter-119, Sca-1, c-Kit, Mac-1, CD3, CD4, CD8, CD24, GR-1 (Pharmingen).

Autopsy, histopathology, and immunohistochemistry

Animals were autopsied as needed and all tissues were examined regardless of their pathological status. Normal and tumor tissue samples were fixed in $10 \%$ buffered formalin and embedded in paraffin. Sections (4-5 $\mu \mathrm{m}$ ) were stained with hematoxylin and eosin according to standard protocols. Representative samples were selected for immunohistochemical analysis of GM-CSFR $\alpha$ and Ki-67 expression. For GM-CSFR $\alpha$ immunohistochemistry, liver sections from wild-type and $\mathrm{Mzf1}^{-/-}$mice were dewaxed in xylene, rehydrated through a graded series of alcohols, treated with $1 \% \mathrm{H}_{2} \mathrm{O}_{2}$ in PBS, pretreated with $0,01 \mathrm{M}$ citric acid, microwaved for $15 \mathrm{~min}$, and incubated with anti-mouse GM-CSFR $\alpha$ antibody (Santa Cruz), overnight at $4^{\circ} \mathrm{C}$. Positive signals were developed with diaminobenzidine (DAB) substrate using the avidin-biotin-peroxidase system and slides were counterstained with hematoxylin. Immunohistochemical analysis of Ki-67 (Novocastra) expression was performed on paraffin sections after dewaxing and rehydration through a graded series of alcohols, treatment with $1 \% \mathrm{H}_{2} \mathrm{O}_{2}$, and incubated overnight at $4^{\circ} \mathrm{C}$ with the antiKi67. Positive signals were developed using DAB as mentioned above for GM-CSFR $\alpha$ staining. Immunohistochemical analysis was performed also for the following primary antibodies B220, CD45, TER119, CD34 (Pharmingen), CD3, and S100 (Dako), as described previously (CordonCardo and Richon 1994).

In vitro $B M$ culture

To score for BFU-E, CFU-GM, and CFU-GEMM, BM cultures from $\mathrm{Mzf1}^{-/-}$and wild-type mice were carried out by plating $5 \times 10^{4}$ cells in 1 $\mathrm{mL}$ of $1 \%$ methylcellulose Iscove's modified Dulbecco medium (IMDM) with $30 \% \mathrm{FBS}, 1 \mathrm{U} / \mathrm{mL}$ recombinant human erythropoietin (EPO), $5 \%$ $(\mathrm{v} / \mathrm{v})$ pokeweed mitogen mouse spleen cell-conditioned medium (PWMSCM), $50 \mathrm{ng} / \mathrm{mL}$ recombinant murine steel factor, and $0.1 \mathrm{mM}$ hemin at $5 \% \mathrm{CO}_{2}$ and lowered $5 \% \mathrm{O}_{2}$. Colonies were counted after $7 \mathrm{~d}$ of incubation. A total of $10 \mathrm{Mzf1^{-/- }}$ and 10 control wild-type syngeneic littermates, sex and age (12-week-old) matched were analyzed in two independent experiments.

Cycling status of hemopoietic progenitor cells

The proportion of each progenitor cell type in DNA synthesis (S phase of the cell cycle) was estimated by means of the high-specific-activity (20 $\mathrm{Ci} / \mathrm{mM})\left[{ }^{3} \mathrm{H}\right]$ thymidine kill technique, which is based on calculation of the reduction in the number of colonies formed in vitro after pulse exposure of cells for $20 \mathrm{~min}$ to $\left[{ }^{3} \mathrm{H}\right]$ thymidine, as compared with control nonradioactive thymidine (Maze et al. 1992). $\mathrm{Mzf1}^{-/-}$and control wildtype syngeneic littermates sex and age (12-week-old) matched were used for this analysis.

Long-term BM culture

Cells were plated at low density, $10^{5}$ per $1.5 \mathrm{~mL}$ of IMDM containing $20 \%$ FBS, $20 \mu \mathrm{M} \beta$-mercaptoethanol, $100 \mathrm{ng} / \mathrm{mL}$ murine Steel factor, 100 $\mathrm{ng} / \mathrm{mL}$ Flt3-ligand, $50 \mathrm{ng} / \mathrm{mL} \mathrm{IL}-6$, and antibiotics (Pen-Strep-Ampho, $\mathrm{BRL})$. Independent cultures were set up for each mouse and $1 \mathrm{~mL}$ of medium was removed weekly. Progenitor assays were carried out as described above, on a weekly base for $4 \mathrm{wk}$, with $10^{4}$ cells taken from these cultures. $\mathrm{Mzf1}^{-1-}$ and control wild-type syngeneic littermates sex and age (12-16-week-old) matched were used for this analysis.

Western blot and Real-time PCR (TaqMan)

Analysis of Myb expression was performed by Western blot on whole-cell protein extracts prepared at different time points $(0,2,4$, and $6 \mathrm{~d})$ from BM cultures treated with IL3 + SC-F + GM-CSF using with the 5E antic-Myb specific antibody (Sleeman 1993; courtesy of Joe Lipstick, Stanford University School of Medicine, CA). TaqMan analysis of c-Myb expres- 
sion was performed on total RNA extracted from sorted c-kit/Fc $\gamma$ III/II Receptor double positive myeloid progenitors using an ABI Prism 7700 Sequence Detection System (PE Biosystems). Sequences of primers and probe for mouse c-Myb are as follows: sense 5'-CGCCGAAGCACA AAACATC-3'; antisense 5'-GGGACGTTGACTATATTAACATGCA3'; 6FAM-CCAGTCACGTTCCCTATCCTGTCGCA-TAMRA. Early myeloid progenitors from a total of four $\mathrm{Mzf1}^{-/-}$and four control wildtype syngeneic littermates, sex and age matched were analyzed in four independent experiments.

Apoptosis analysis by TUNEL and Annexin $V$

Detection analysis of apoptosis was performed by Annexin V staining according to published procedures (Rego et al. 2001) in tricolor flow cytometry on the following BM cellular populations from $\mathrm{Mzf1}^{-/-}$and control littermates: CD $34^{+} / \mathrm{lin}^{-}$or $\mathrm{lin}^{+}$; CD $34^{+} / \mathrm{Sca}-1^{+}$; CD $34^{+} / \mathrm{c}-\mathrm{kit}{ }^{+}$. Fluorochrome-conjugated antibodies for flow cytometry were: CD34, c-kit, and Sca-1 (Pharmingen). B220, CD3, Mac-1, Ter119, and Gr-1 antibodies (Pharmingen), all PE conjugated, were pooled as "lineage positive" $\left(\operatorname{lin}^{+}\right)$ to subdivide CD $34^{+}$cells in lin $^{+}$and lin $^{-}$subsets. FACS-sorted populations were also collected and spun on glass slides, fixed in $4 \%$ buffered formalin and stained by in situ TUNEL assay according to published protocols (Di Cristofano et al. 2001).

Transplantation

Leukemic cells were transplanted essentially as described previously

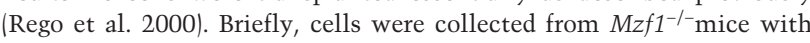
liver neoplasia and leukemia as well as wild-type controls either by passing liver cells through a strainer to remove hepatocytes, or by flowsorting CD45-expressing myeloid cells from the liver or the spleen. Blasts $\left(2 \times 10^{7}\right)$ were injected intraperitoneally into $129 / \mathrm{Sv}$ syngeneic sublethally irradiated mice (500 Gy), in triplicate, immediately after collection. The recipient mice were followed up for tumor growth on the site of injection and for leukemia development.

\section{Acknowledgments}

We thank J. Lipsick and J.F. Morris for materials. We also thank M. Barna, P. Clarck, J. Costoya-Puente, M. Jiao, S. Kalantry, L. Longo, L. Luzzatto, T. Merghoub, R. Notaro, E. M. Rego, K. Scotto, and V. Soares for assistance and advice. These studies were partially supported by the Associazione Italiana per la Ricerca sul Cancro (AIRC) (M.G.), and by National Institutes of Health grants to H.E.B., R.H., and P.P.P. R.H. and P.P.P. are Scholars of the Leukemia and Lymphoma Society (formerly known as the Leukemia Society of America).

The publication costs of this article were defrayed in part by payment of page charges. This article must therefore be hereby marked "advertisement" in accordance with 18 USC section 1734 solely to indicate this fact.

\section{References}

Barna, M., Hawe, N., Niswander, L., and Pandolfi, P.P. 2000. Plzf regulates limb and axial skeletal pattering. Nat. Genet. 25: 166-172.

Bavisotto, L., Kaushansky, K., Lin, N., and Hromas, R. 1991. Antisense oligonucleotides from the stage-specific myeloid zinc finger gene MZF-1 inhibit granulopoiesis in vitro. J. Exp. Med. 174: 1097-1101.

Cordon-Cardo, C. and Richon, V.M. 1994. Expression or the retinoblastoma protein is regulated in normal human tissue. Am. J. Pathol. 144: 500-510.

Di Cristofano, A., De Acetis, M., Koff, A., Cordon-Cardo, C., and Pandolfi, P.P. 2001. Pten and p27KIP1 cooperate in prostate cancer tumor suppression in the mouse. Nat. Genet. 27: 222-224.

Harrison, T.R. 1987. The leukemias. In Harrison's principles of internal medicine, 11th ed. (ed. E. Braunwald, K.J. Isselbacher, R.G. Petersdorf, J.D. Wilson, J.B. Martin, and A.S. Fauci), pp. 1545-1589. McGraw-Hill Book Company, New York.

He, L.Z., Tribioli, C., Rivi, R., Peruzzi, D., Pelicci, P.G., Soares, V., Cattoretti, G., and Pandolfi, P.P. 1997. Acute leukemia with promyelocytic features in PML/RAR $\alpha$ transgenic mice. Proc. Natl. Acad. Sci. 94: 5302-5307.

He, L.Z., Bhaumik, M., Tribioli, C., Rego, E.M., Ivins, S., Zelent, A., and Pandolfi, P.P. 2000. Two critical hits for promyelocytic leukemia. Mol. Cell 6: 1131-1141.
Hoffman, S.M., Hromas R., Amemiya, C., and Mohrenweiser H.W. 1996 The location of MZF-1 at telomere of human chromosome 19q makes it vulnerable to degeneration in aging cells. Leuk. Res. 20: 281-283.

Hromas, R., Collins, S.J., Hickstein, D., Raskind, W, Deaven, L.L., O'Hara, P., Hagen, F.S., and Kaushansky, K. 1991. A retinoic acidresponsive human zinc finger gene, MZF-1, preferentially expressed in myeloid cells. J. Biol. Chem. 266: 14183-14187.

Hromas, R., Davis, B., Rauscher, F.J., Klemsz, M., Tenen, D., Hoffman, S., $\mathrm{Xu}, \mathrm{D}$., and Morris, J.F. 1996. Hematopoietic transcriptional regulation by the myeloid zinc finger gene, MZF-1. Curr. Top. Microbiol. Immunol. 211: 159-164.

Look, A.T. 1997. Oncogenic transcription factors in the human acute leukemias. Science 278: 1059-1064.

Maze, R., Sherry, B., Kwon, B.S., Cerami, A., and Broxmeyer, H.E. 1992 Myelosuppressive effects in vivo of purified recombinant murine macrophage inflammatory protein-1 alpha. J. Immunol. 149: 10041009.

Morris, J.F., Hromas, R., and Rauscher, F.J. 1994. Characterization of the DNA-binding properties of the myeloid zinc finger protein MZF1: Two independent DNA-binding domains recognize two DNA consensus sequences with a common G-rich core. Mol. Cell. Biol. 14: $1786-1795$.

Morris, J.F., Rauscher, F.J., Davis, B., Klemsz, M., Xu, D., Tenen, D., and Hromas, R. 1995. The myeloid zinc finger gene, MZF-1, regulates the CD34 promoter in vitro. Blood 86: 3640-3647.

Murai, K., Murakami, H., and Nagata, S. 1997. A novel form of the myeloid-specific zinc finger protein (MZF-2). Genes Cells. 2: 581591.

. 1998. Myeloid-specific transcriptional activation by murine myeloid zinc-finger protein 2. Proc. Natl. Acad. Sci. 95: 3461-3466.

Perrotti, D., Melotti, P., Skorski, T., Casella, I., Peschle, C., and Calabretta, B. 1995. Overexpression of the zinc finger protein MZF1 in hibits hematopoietic development from embryonic stem cells: Correlation with negative regulation of CD34 and c-myb promoter activity. Mol. Cell. Biol. 15: 6075-6087.

Rego, E., He, L.Z., Warrell, Jr, R.P., Wang, Z.G., and Pandolfi, PP. 2000 Retinoic acid (RA) and As2O3 treatment in transgenic models of acute promyelocytic leukemia (APL) unravel the distinct nature of the leukemogenic process induced by the PML-RAR $\alpha$ and PLZFRAR $\alpha$ oncoproteins. Proc. Nat1. Acad. Sci. 97: 10173-10178.

Rego, E., Wang, Z., Peruzzi, D., He, L.Z., Cordon-Cardo, C., and Pandolfi, P.P. 2001. Role of promyelocytic leukemia (pml) protein in tumor suppression. J. Exp. Med. 193: 521-530.

Sander, T.L., Haas, A.L., Peterson, M.J., and Morris, J.F. 2000. Identification of a novel SCAN box-related protein that interacts with MZF1B. The leucine-rich scan box mediates hetero- and homoprotein associations. J. Biol. Chem. 275: 12857-12867.

Shivdasani, R.A. and Orkin, S.H. 1996. The transcriptional control of hematopoiesis. Blood 87: 4025-4039.

Sleeman, J.P. 1993. Xenopus A-myb is expressed during early spermatogenesis. Oncogene 8:1931-1941.

Tybulewicz, V.L.J., Crawford, C.E., Jackson, P.K., Bronson, R.T., and Mulligan, R.C. 1991. Neonatal lethality and lymphopenia in mice with homozygous disruption of the c-abl proto-oncogene. Cell 65: 1153-1163. 


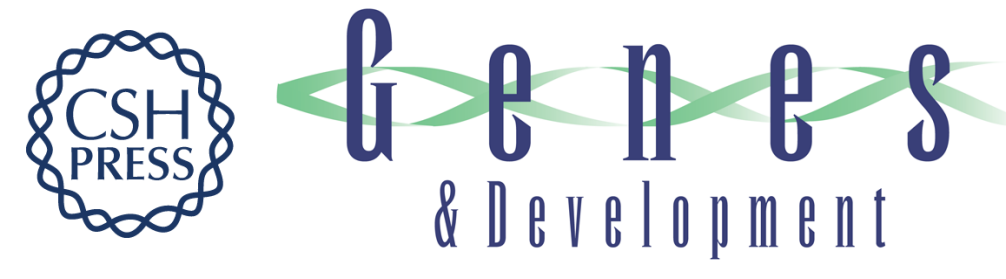

\section{Mzf1 controls cell proliferation and tumorigenesis}

Mirella Gaboli, Paraskevi A. Kotsi, Carmela Gurrieri, et al.

Genes Dev. 2001, 15:

Access the most recent version at doi:10.1101/gad.902301

References This article cites 22 articles, 13 of which can be accessed free at: http://genesdev.cshlp.org/content/15/13/1625.full.html\#ref-list-1

License

Email Alerting Receive free email alerts when new articles cite this article - sign up in the box at the top Service right corner of the article or click here.

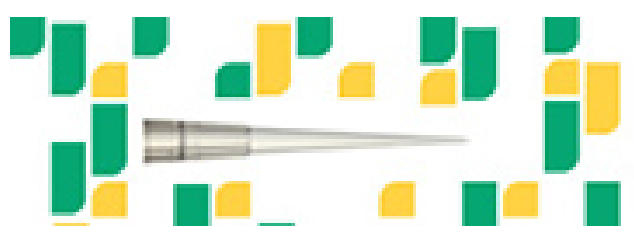

Focused on your science. 